\title{
Stimulating Photo Curation on Smartphones
}

\author{
Xenia Zürn \\ University of Technology Sydney, \\ Eindhoven University of Technology \\ z.xenia@gmail.com \\ Saskia Bakker \\ University of Technology Sydney, \\ Eindhoven University of Technology \\ s.bakker@tue.nl
}

\author{
Mendel Broekhuijsen \\ Eindhoven University of Technology, \\ University of Technology Sydney \\ mendel@mendeldesign.nl \\ Annemarie Zijlema \\ University of Technology Sydney, \\ Eindhoven University of Technology \\ afzijlema@hotmail.com
}

\author{
Doménique van Gennip \\ University of Technology Sydney, \\ Eindhoven University of Technology \\ domenique.vangennip@uts.edu.au \\ Elise van den Hoven \\ University of Technology Sydney, \\ Eindhoven University of Technology, \\ University of Dundee \\ elise.vandenhoven@uts.edu.au
}

\begin{abstract}
Personal photo collections have grown due to digital photography and the introduction of smartphones, and photo collections have become harder to manage. Deleting photos appears to be difficult and the task of curation is often perceived as not enjoyable. The lack of curation can make it harder to retrieve photos when people need them for various reasons, such as individual reminiscing, shared remembering or self-presentation. In this study we investigate how we can stimulate people to organise their photo collections on their smartphones. Ten participants evaluated and qualitatively compared four applications with different characteristics regarding voting on and deleting photos. We found that voting on photos is easier and more enjoyable in comparison to deleting photos, that participants showed reminiscence while organising, that deleting can be frustrating, that participants have different preferences for sorting and viewing photos and that voting could make deleting and retrieving easier.
\end{abstract}

\section{CCS CONCEPTS}

- Information systems Users and interactive retrieval • Humancentered computing Interaction design $\bullet$ Human-centered computing $\sim$ Smartphones

\section{KEYWORDS}

Photo Curation, Smartphone, Interaction Design, Design Research

\section{INTRODUCTION}

Over the past decades, personal photo collections have grown in size due to the rise of digital photography and reduced cost of storage $[1,18,30]$. The way people interact with personal photos has changed since the introduction of digital photography and especially camera phones that further increased the size of personal photo collections [14,22-24]. Due to their ever-expanding volume, it is increasingly difficult to manage personal photo collections [30]. People find deleting their photos difficult and would rather keep most of them [e.g.,15,18,29]. Moreover, the sheer number of photos makes curation a daunting task. People seem to be discouraged to curate their collections due to a perceived lack of expertise and time to manage a large collection $[18,28,30]$. People also suffer from a lack of motivation because curation is considered to be a chore, which is neither creative nor rewarding [26]. As a consequence, people experience difficulties retrieving valuable photos from their collections [30].

The study of people's curation activities of personal photos, as presented in this paper, is part of the research area 'Personal Information Management' (PIM). PIM involves the study of managing personal information, which besides photos includes emails, bookmarks and personal files [e.g.,3,11,17], and differs from other information management practices in that people have to curate for their future selves [2]. The curation of personal information is a pro-active activity and therefore often postponed or ignored [16].

This paper discusses a comparison study of four mobile applications that help to prune one's photo collections. We focus on the smartphone because for many people it has become their primary device for photo activities [14,22-24]. However, curating on smartphones is still rare and open for further investigation [33]. Following Broekhuijsen et al. in their PhotoUse model [5], we focus on organising (i.e., tagging, moving, categorizing, naming, captioning, archiving, and deleting) within the broader activity of curation. The present study took the challenge to leverage interaction design as a means to stimulate people to organise their 
photo collections on smartphones. Hereby, we investigate how voting on photos rather than deleting these could stimulate the organisation of personal collections.

\section{RELATED WORK}

This section describes research from Human-Computer Interaction and Computer-Supported Collaborative Work that relates to interaction with and curation of digital photo collections using mobile devices.

In a study by Ott, Hebecker, and Wakes [25] three interaction concepts, aimed at engaging people with their personal digital photos, were evaluated. The authors had difficulty in identifying specific design factors that focus on entertainment and play. While their study focused on management and presentation of personal digital photographs, it did not address the user experience of organising photos as we do in this paper.

There are a few examples that focus on group curation, but do not address the organisation of personal collections [e.g.,6,21,27]. GrayArea by Bergman et al. [1] is an example that is more closely related to the practices of deleting unwanted files in personal digital collections. It introduces a new kind of deleting of files called demoting, temporary hiding it from view instead of throwing it away, using the metaphor of storing physical items in an attic. Although not intended for just photos, it helps us to reflect on the way we interact with digital information. Demoting can be seen as organising in phases because it formalises a process where people do not delete photos right away, but place them in a digital attic for later disposal.

The related work makes clear that the design of photo use devices leaves room to explore how we can decrease mental effort of making organisational decisions while organising our personal digital collections. Because organising is usually perceived as a daunting but relatively unrewarding task, we believe that applications that support organising should not only reduce the mental effort of the activity but also enhance the experience itself. Organising photos is usually performed during leisure time and we argue that an efficient and pleasant experience may motivate people to drive improvements in the organisational state of personal photo collections.

\section{STUDY}

This study qualitatively compares four smartphone applications to explore how interaction design elements support and stimulate the organisation of personal photos. As described above, existing applications are limited in use because there is significant mental effort $[15,18,29]$ in organising photo collections and a lack of enjoyment in the organising task itself [26]. Therefore, we compare two existing smartphone applications designed for viewing, selecting, and deleting one's photos and complement these with two applications designed to decrease effort and to increase enjoyment while organising.

\section{Voting application}

Decisions to delete photos are hard because these are irreversible, thus we reason that merely voting on photos could make the organisation task easier. We designed the Voting application in which users vote photos up or down using swiping gestures. Because swiping gestures can increase enjoyment and can positively influence reuse intentions [7,9], we implemented the swiping gesture for decision making in the Voting application. The Voting application has some conceptual overlap with the Phototriage application by Drucker et al. [10]. In the Voting application (Figure 1A), participants can swipe right or press the 'up arrow button' to vote photos up (Figure 1C) and swipe left or press the 'down arrow button' to vote photos down (Figure 1B). Photos are 'down voted' or 'up voted' instead of kept or deleted.

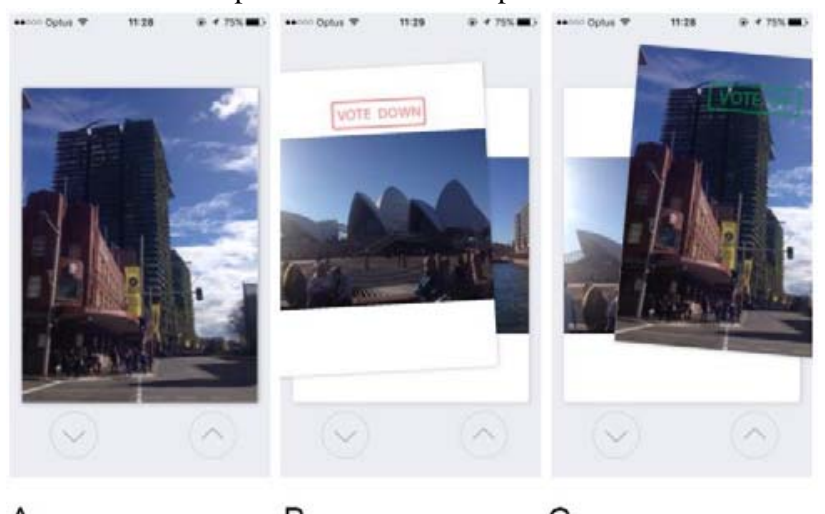

A

B

C

Figure 1. Upvoting and downvoting photos with the Voting application. 1A: main view with downvote and upvote buttons; 1B: downvote a photo; 1C: upvote a photo.

\section{Dilemma Voting application}

Showing digital content in a random manner can evoke serendipity [19]. People are willing to give up direct control over what they see in return for the enjoyment of surprising interactions [31]. Frohlich et al. [13] suggest that serendipitous browsing should be stimulated since the most powerful instances of reinterpretation occur after seeing a photo in an unexpected manner. They also suggest that seeing a photo in an unexpected juxtaposition with other photos might trigger reinterpretation. To see whether random display of photos in unexpected juxtapositions while organising can increase enjoyment, we designed the Dilemma Voting application. This application (Figure 2A) asks a participant to choose between two random photos by tapping the most valued photo. While pressing a photo, a 'flash dot' is shown on the pressed photo (Figure 2B). After that, two new random photos are shown. In order to decrease mental effort, photos are 'downvoted' or 'upvoted' instead of kept or deleted. 


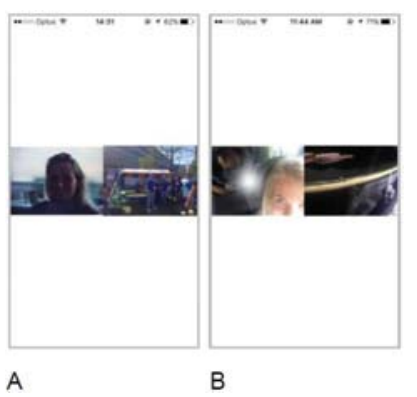

Figure 2. Upvoting and downvoting photos with the Dilemma Voting application. 2A: main view; 2B: showing a flash dot (in this case on the left photo) when upvoting a photo by touching it.

Next to the two designed apps we described above, we used two commercially available applications during the study: 1 ) the default Photos application on Apple iOS9, allowing a comparison to the status quo, and 2) the application Pare Down [12]. The latter is very similar to our own Voting application, however, instead of voting photos up or down, photos are kept or deleted.

As part of this study, we invite participants to comment on an idea for the visualisation of the voting data, which we call the Voted Gallery, because only voting on photos does not make a photo collection more manageable, if the voting metadata is not put to good use.

\section{Voted Gallery}

The Voted Gallery has features similar to a typical camera roll view on smartphones, showing thumbnails of the photos in chronological order. People prefer this way of viewing photos on their smartphones because it provides an overview and context for the photos $[28,33]$. Moreover, other studies found that people use context (i.e. event-based browsing) and thumbnail view in order to retrieve photos $[18,30]$ and that this way of viewing can help to make decisions on what to keep [32]. The Voted Gallery differs from the camera roll view because it displays thumbnails of upvoted photos bigger and downvoted photos smaller. The size of the thumbnails correspond with the number of upvotes or downvotes, which could be helpful for people to find the valued photos among their collections, also echoing Petrelli and Whittaker's suggestion that a "distinction between an implicit favourite and the rest of a collection could promote new and engaging ways of revisiting” [26, p.167].

\section{Participants}

Participants were recruited from the personal and professional network of the researchers, and invited to participate via email. They were required to own a smartphone containing at least 600 digital photos. Ten people participated (7 women), aged 22-55 $(M=28.9, S D=8.9)$. Six participants were native English speakers and the other four had comparable language skills.

\section{Procedure}

Participants were asked to bring 600 most recent (unsorted) photos from their smartphone photo collection. After participants signed a consent sheet, a subset of 150 photos were selected by the researcher and uploaded to the smartphone that was used for this study (Apple iPhone 5). After a short explanation of the smartphone application, the participants used the smartphone application for ten minutes, or until all photos were sorted. This process was repeated for all four applications, using a new set of 150 photos from the participant for each application. For each participant, the order of using the applications was randomised. Afterwards, a semistructured interview probed participants for their experiences while using the applications and asked them to compare the applications. To stimulate the conversation but not miss out on unprompted ideas, we introduced the Voted Gallery after ranking the applications and after probing participants for the perceived usefulness of voting applications. Each session lasted for about 1.5 hours. Interviews were transcribed and selectively coded. We used thematic analysis to interpret the data and find common themes [4].

\section{FINDINGS}

Our analysis led to themes regarding the experience of voting and deleting, game elements, reminiscence, sorting preferences, combining voting and deleting, and how people's associations influence curation practices. In this section we will elaborate on these themes.

As expected, we found that voting on photos is easier and more enjoyable in comparison to deleting photos. Participants regarded deleting photos as something practical that needs to be done and therefore not that enjoyable. Voting on photos is regarded more positive. Also, as P3 and P7 illustrate, the limited impact of errors in voting makes that voting on photos is more enjoyable in comparison to deleting photos: "I think deleting is something practical, that's something you do when [...] memory is full, I have to delete photos. Upvoting is something you do because you want to do it and not because it's practical." (P7). And P3: "[The Voting application] I found sort of enjoyable, [Pare Down] I also found enjoyable [...] but it felt like it was a bit sort of cutthroat because I was actually deleting things."

A more enjoyable organising experience was also supported through the game elements of curiosity, discovery and challenge [20]. Participants' curiosity arose while using the swiping applications and the Dilemma Voting application while exploring their photo collection. Challenge, "the experience of suspense transforming into ecstatic release once some obstacle is overcome" [20, p.3], was observed by participants when using the Dilemma Voting application to choose between two photos. As P10 said: "Quick, show me what's next, what's next, what's next! It's good fun," and P1: "I also enjoyed picking, even though it was frustrating."

We noted that participants showed signs of positive reminiscence during and after using the applications. This implies that organising photos is not only a way to improve reminiscence due to a better organised photo collection but that reminiscence also takes place 
while organising: "I enjoyed the experience of [using the Voting application]. I found it fun, I really liked the fact that it allowed you to revisit those memories and I think that that in itself is a really nice experience, I want to do it again," (P3).

Nine participants considered the standard application to be the least enjoyable to use. They felt too much of a barrier to delete photos, which annoyed or even frustrated the participants: "It really annoys me that when you bin it and then it asks you 'are you sure you want to delete this photo?' again. So it's twice you have to hit it," (P2). Some participants liked the Dilemma Voting application for its approach to photo sorting, since one photo provides the reference for the other photo. However, others found the Dilemma Voting application not useful for sorting photos because it did not cater to their preferred way of sorting photos. This led to suggestions that the Dilemma Voting application would be more useful if it only rates photos of the same event or category, or to compare duplicates. As P4 put it: "So if I had a hundred photos [of a party] and I compared a lot of them during the night [using the Dilemma Voting application] and after I would have them ranked, I would create an album with that and post it on Facebook or Instagram." Voting between two photos indeed posed a dilemma at times: "Sometimes I felt like I was having to leave a very nice photo behind and sometimes I was having to pick a not so nice photo," (P3). Participants compared the content of the photos instead of comparing the quality of the photos. This also highlights that the photos were seen as representative of participants' associations: "This isn't fair. I've got a beautiful family moment [versus] my boyfriend looking like a dog... I can't compare them!” (P1).

Several participants considered voting on photos prior to deleting photos valuable. They saw how the Voting application in combination with the Voted Gallery could help them to make deleting decisions. Moreover, three participants mentioned that they would be interested to see how they would rank their photos over time: "Then I can actually see over time which photos I actually do like as opposed to me telling myself I like them. It's like the coat hanger trick when you're sorting through all your extra clothes. You hang it backwards and when you wear it, you turn it back the normal way. So at the end, you can actually see which clothes you wear and [which to] get rid of," (P1). Furthermore, participants mentioned that the Voted Gallery could help them to find photos and show photos to friends. This is in line with other studies suggesting that creating an attractive presentation of one's favourite photos in order to share them is a key motivation to organise photos $[25,28]$.

\section{DISCUSSION \& CONCLUSION}

The present study indicates that people have different desires at different moments regarding the organisation of photos on their smartphone. For example, some people preferred the Dilemma Voting application and others the Voting application in order to organise their photos. People would also like to view photos in the Voted Gallery differently, since they would like to use it for different reasons (e.g., deleting versus showing photos to others). This is also in line with a study by Cosley et al. in which users pointed out that they would like to have control over when and how they view memory triggers [6]. Furthermore, we noticed that participants reminisce while organising and that some participants were quite frustrated about deleting photos with the default iOS application. Thus, we believe that multiple options to organise photo collections should be provided in order to fit more users. For example, the reminiscence experience and its occurrence during organising should be considered in future designs.

In this study, participants brought a copy of their photos and might therefore not have been hesitant to delete their photos during the organising task. Although we asked whether they would perform differently on their own phone or without a backup, the current findings are indicative only. Participants used the four applications with a new set of photos for each application. We did this because the participants could experience serendipity in each of the applications and would not get bored with viewing each set of photos four times. However, the photos in each set differ and thus might evoke different emotions, which could have influenced the experience of using the applications.

In this study we investigated the use of four applications within a lab setting, with each application only used for about ten minutes. We gained insights about which aspects of the applications could stimulate organisation of personal photo collections. However, the only way to validate the theoretical assumptions and test if these aspects really stimulate organisation, the applications would need to be developed further and tested long-term. It would be worthwhile to evaluate an application that uses a voting system in combination with our Voted Gallery concept to see if our design assumptions still hold in everyday use and to what extent it will stimulate organisation photo collections. For example, voting on photos might increase people's awareness of the photos they do not value enough to keep, which could reduce the perceived effort of deleting those photos and contribute to the overall value of their photo collections.

The results presented in this paper support using a voting system for organising our personal digital photo collections on smartphones. The results indicate that a voting system could support the deleting of photos and also could improve retrieving of our photo collections. Furthermore, we found that a voting system, introduction of a Voted Gallery, implementation of the swiping gesture and randomness in displaying photos can increase the enjoyment of the organising practice and therefore could stimulate curation of our personal digital photo collections. Despite the small sample size, we believe our findings provide valuable insights for the design of novel photo organising applications on smartphones.

\section{ACKNOWLEDGMENTS}

We thank all the participants for their contribution to our study. This research was supported by STW VIDI grant number 016.128.303 of The Netherlands Organisation for Scientific Research (NWO), awarded to Elise van den Hoven. The study was approved by the UTS Research Ethics Committee under REF ETH16-855.

\section{REFERENCES}


[1] Ofer Bergman, Simon Tucker, Ruth Beyth-Marom, Edward Cutrell, and Steve Whittaker. 2009. It's Not That Important: Demoting Personal Information of Low Subjective Importance Using GrayArea. In Proceedings of the SIGCHI Conference on Human Factors in Computing Systems (CHI '09), 269-278. DOI: https://doi.org/10.1145/1518701.1518745

[2] Ofer Bergman and Steve Whittaker. 2016. The Science of Managing our Digital Stuff. MIT Press, Cambridge, MA. DOI: https://doi.org/10.1002/asi.23870.

[3] Richard Boardman and M. Angela Sasse. 2004. "Stuff Goes into the Computer and Doesn't Come Out" A Cross-tool Study of Personal Information Management. In Proceedings of the SIGCHI conference on Human factors in computing systems (CHI'04). ACM Press, Vienna, Austria, 583-590. DOI: https://doi.org/10.1.1.115.3372.

[4] Virginia Braun and Victoria Clarke. 2006. Using thematic analysis in psychology. Qualitative Research in Psychology 3, 2: 77-101. DOI: https://doi.org/10.1191/1478088706qp063oa

[5] Mendel Broekhuijsen, Elise van den Hoven, and Panos Markopoulos. 2017. From PhotoWork to PhotoUse: exploring personal digital photo activities. Behaviour \& Information Technology 36, 7: 754-767. DOI: https://doi.org/10.1080/0144929X.2017.1288266

[6] Frederik Brudy, Steven Houben, Nicolai Marquardt, and Yvonne Rogers. 2016 CurationSpace: Cross-Device Content Curation Using Instrumental Interaction. In Proceedings of the 2016 ACM International Conference on Interactive Surfaces and Spaces (ISS '16), 159-168. DOI: Surfaces and Spaces (ISS

[7] Ben C F Choi, Samuel N Kirshner, and Yi Wu. 2016. Swiping vs. Scrolling in Mobile Shopping Applications. In HCI in Business, Government, and Organizations: eCommerce and Innovation, 177-188.

[8] Dan Cosley, Kathy Akey, Brian Alson, Jonathan Baxter, Mark Broomfield, Soyoung Lee, and Chethan Sarabu. 2009. Using Technologies to Support Reminiscence. In Proceedings of the 23rd British HCI Group Annual Conference on People and Computers: Celebrating People and Technology (BCS-HCI '09), 480-484.

[9] Xue Dou and S Shyam Sundar. 2016. Power of the Swipe: Why Mobile Websites Should Add Horizontal Swiping to Tapping, Clicking, and Scrolling Interaction Techniques. International Journal of Human-Computer Interaction 32, 4: 352362. DOI: https://doi.org/10.1080/10447318.2016.1147902

[10] Steven Drucker, Curtis Wong, Asta Roseway, Steve Glenner, and Steve De Mar. 2003. Photo-triage: Rapidly annotating your digital photographs. Tech. rep., Microsoft Research Technical Report, MSR-TR-2003-99.

[11] David Elsweiler and Ian Ruthven. 2007. Towards task-based personal information management evaluations. In Proceedings of the 30th annual international ACM SIGIR conference on Research and development in information retrieval. ACM Press, Amsterdam, the Netherlands, 23-30. DOI: https://doi.org/10.1145/1277741.1277748.

[12] Fly Soup. 2014. Pare Down. Retrieved from https://itunes.apple.com/nl/app/pare-down-photo-albumeditor/id914281628?mt=8

[13] David M Frohlich, Steven Wall, and Graham Kiddle. 2013. Rediscovery of Forgotten Images in Domestic Photo Collections. Personal Ubiquitous Comput. 17, 4: 729-740. DOI: https://doi.org/10.1007/s00779-012-0612-4

[14] Lisa Gye. 2007. Picture This: the Impact of Mobile Camera Phones on Personal Photographic Practices. Continuum 21, 2: 279-288. DOI: https://doi.org/10.1080/10304310701269107

[15] Nancy Van House and Elizabeth F Churchill. 2008. Technologies of memory: Key issues and critical perspectives. Memory Studies 1, 3: 295-310. DOI: https://doi.org/10.1177/1750698008093795

[16] William Jones. 2007. Personal Information Management. Annual Review of Information Science and Technology (ARIST), 41, 1, 453-504. DOI: https://doi.org/10.1002/aris.2007.1440410117.

[17]William Jones, Ammy Jiranida Phuwanartnurak, Rajdeep Gill and Harry Bruce. 2005. Don't take my folders away! Organizing personal information to get things done. CHI'05 extended abstracts on Human factors in computing systems. ACM Press, Portland, Oregon USA, 1505-1508. DOI: https://doi.org/10.1145/1056808.1056952.

[18] David Kirk, Abigail Sellen, Carsten Rother, and Ken Wood. 2006. Understanding Photowork. In Proceedings of the SIGCHI Conference on Human Factors in $\begin{array}{llll}\text { Computing Systems (CHI '06), 761-770. DOI: } & \end{array}$ https://doi.org/10.1145/1124772.1124885

[19] Tuck Leong, Steve Howard, and Frank Vetere. 2008. Choice: Abidcating or Exercising? In Proceedings of the SIGCHI Conference on Human Factors in Computing Systems (CHI '08), 715-724. DOI: https://doi.org/10.1145/1357054.1357168

[20] Craig A Lindley and Charlotte C Sennersten. 2008. Game Play Schemas: From Player Analysis to Adaptive Game Mechanics. Int. J. Comput. Games Technol. 2008: 8:1--8:7. DOI: https://doi.org/10.1155/2008/216784

[21] Andrés Lucero, Jussi Holopainen, and Tero Jokela. 2011. Pass-them-around: Collaborative Use of Mobile Phones for Photo Sharing. In Proceedings of the
SIGCHI Conference on Human Factors in Computing Systems (CHI '11), 17871796. DOI: https://doi.org/10.1145/1978942.1979201

[22] Effie Le Moignan, Shaun Lawson, Duncan A Rowland, Jamie Mahoney, and Pam Briggs. 2017. Has Instagram Fundamentally Altered the "Family Snapshot”? In Proceedings of the 2017 CHI Conference on Human Factors in Computing Systems (CHI '17), 4935-4947. DOI: https://doi.org/10.1145/3025453.3025928

[23] Susan Murray. 2008. Digital Images, Photo-Sharing, and Our Shifting Notions of Everyday Aesthetics. Journal of Visual Culture 7, 2: 147-163. DOI: https://doi.org/10.1177/1470412908091935

[24] Virginia Nightingale. 2007. The Cameraphone and Online Image Sharing Continuum 21, 2: 289-301. DOI: https://doi.org/10.1080/10304310701278157

[25] Claudia Ott, Ralf Hebecker, and Sarah Wakes. 2012. Picture the Space: Three Concepts for Management and Presentation of Personal Digital Photographs. In Proceedings of the 13th International Conference of the NZ Chapter of the ACM's Special Interest Group on Human-Computer Interaction (CHINZ '12), 1-8. DOI: https://doi.org/10.1145/2379256.2379258

26] Daniela Petrelli and Steve Whittaker. 2010. Family Memories in the Home: Contrasting Physical and Digital Mementos. Personal Ubiquitous Comput. 14, 2: 153-169. DOI: https://doi.org/10.1007/s00779-009-0279-7

[27] Martin Porcheron, Andrés Lucero, and Joel E Fischer. 2016. Co-curator: Designing for Mobile Ideation in Groups. In Proceedings of the 20th International Academic Mindtrek Conference (AcademicMindtrek '16), 226-234. DOI: https://doi.org/10.1145/2994310.2994350

[28] Kerry Rodden and Kenneth R Wood. 2003. How Do People Manage Their Digital Photographs? In Proceedings of the SIGCHI Conference on Human Factors in Computing Systems (CHI '03), 409-416. DOI: https://doi.org/10.1145/642611.642682

[29] Steve Whittaker. 2013. Personal information management: From information consumption to curation. Annual Review of Information Science and Technology 45, 1: 1-62. DOI: https://doi.org/10.1002/aris.2011.1440450108

[30] Steve Whittaker, Ofer Bergman, and Paul Clough. 2010. Easy on That Trigger Dad: A Study of Long Term Family Photo Retrieval. Personal Ubiquitous Comput. 14, 1: 31-43. DOI: https://doi.org/10.1007/s00779-009-0218-7

[31] John Williamson and Lorna M Brown. 2008. Flutter: Directed Random Browsing of Photo Collections with a Tangible Interface. In Proceedings of the 7th ACM Conference on Designing Interactive Systems (DIS '08), 147-155. DOI: https://doi.org/10.1145/1394445.1394461

[32] Maria K Wolters, Elaine Niven, and Robert H Logie. 2014. The Art of Deleting Snapshots. In CHI '14 Extended Abstracts on Human Factors in Computing Systems (CHI EA '14), 2521-2526. DOI: https://doi.org/10.1145/2559206.2581287

[33] Xenia Zürn, Koen Damen, Fabienne van Leiden, Mendel Broekhuijsen, and Panos Markopoulos. 2018. Photo Curation Practices on Smartphones. In Proceedings of ACE 2017: 14th International Conference on Advances in Computer Entertainment Technology, 406-414. DOI: https://doi.org/10.1007/978-3-319-76270-8_28 